[12] S. I. Niculescu, E. I. Verriest, L. Dugard, and J. M. Dion, "Stability and robust stability of time-delay systems: A guided tour," in Stability and Control of Time-Delay Systems, L. Dugard and E. I. Verriest, Eds. New York: Springer-Verlag, 1998, pp. 1-71.

[13] K. Pyragas, "Continuous control of chaos by self-controlling feedback," Phys. Lett. A, vol. 170, pp. 421-428, 1992.

[14] K. Pyragas and A. Tamaševičius, "Experimental control of chaos by delayed self-controlling feedback," Phys. Lett. A, vol. 180, pp. 99-102, 1993.

[15] K. Pyragas, "Control of chaos via extended delay feedback," Phys. Lett. A, vol. 206, pp. 323-330, 1995.

[16] T. Ushio, "Limitation of delayed feedback control in nonlinear discrete-time systems," IEEE Trans. Circuits Syst. I, vol. 43, pp. 815-816, Sept. 1996.

[17] T. Ushio and S. Yamamoto, "A generalization of delayed feedback control in chaotic discrete-time systems," in Proc. IEEE Conf. Decision Control, 1998, pp. 1946-1950.

[18] W. M. Wonham, "On pole assignment in multi-input controllable linear systems," IEEE Trans. Automat. Contr., vol. AC-12, pp. 660-665, June 1967.

[19] S. Yamamoto, T. Hino, and T. Ushio, "A dynamic delayed feedback controller for chaotic discrete-time systems," in Proc. Amer. Control Conf., 2000, pp. 3358-3359.

\section{Stabilization and Disturbance Rejection for the Beam Equation}

Ömer Morgül

\begin{abstract}
We consider a system described by the Euler-Bernoulli beam equation. For stabilization, we propose a dynamic boundary controller applied at the free end of the system. The transfer function of the controller is a marginally stable positive real function which may contain poles on the imaginary axis. We then give various asymptotical and exponential stability results. We also consider the disturbance rejection problem.
\end{abstract}

Index Terms-Boundary control systems, distributed parameter systems, disturbance rejection, flexible structures, semigroup theory, stability.

\section{INTRODUCTION}

Many mechanical systems, such as spacecraft with flexible attachments, or robots with flexible links, and many practical systems such as power systems, mass transport systems contain certain parts whose dynamic behavior can be rigorously described only by partial differential equations (PDEs). In such systems, to achieve high precision demands, the dynamic effect of the system parts whose behavior are described by PDEs on the overall system has to be taken into account in designing the controllers.

In recent years, boundary control of systems represented by PDEs has become an important research area. This idea is first applied to the systems represented by the wave equation (e.g., elastic strings, cables), see, e.g., [2], [8], and then extended to the beam equations, [3], and to the rotating flexible structures, see [11], [12]. In particular, it has been shown that for a beam which is clamped at one end and is free

Manuscript received May 26, 2000; revised January 22, 2001. Recommended by Associate Editor I. Lasiecka.

The author is with the Department of Electrical and Electronics, Engineering, Bilkent University, 06533 Bilkent, Ankara, Turkey (e-mail: morgul@ bilkent.edu.tr).

Publisher Item Identifier S 0018-9286(01)11109-8. at the other end, a single nondynamic boundary control applied at the free end is sufficient to exponentially stabilize the system, see [3]. This result was then extended for dynamic boundary controllers, see [13]. For more references and technical information on the subject the reader is referred to [10].

In this note, we consider a linear time invariant system which is represented by one-dimensional Euler-Bernoulli beam equation in a bounded domain. We assume that the system is clamped at one end and the boundary control input is applied at the other end. For this system, we propose a finite dimensional dynamic boundary controller. This introduces extra degrees of freedom in designing controllers which could be exploited in solving a variety of control problems, such as disturbance rejection, pole assignment, etc., while maintaining stability. The transfer function of the controller is a proper rational function of the complex variable $s$, and may contain a single pole at $s=0$ and another one $s=j \omega_{1}, \omega_{1} \neq 0$, provided that the residues corresponding to these poles are nonnegative; the rest of the transfer function is required to be a strictly positive real function. Such transfer functions are called marginally stable positive-real (MSPR) functions, see [7]. This type of controllers have been proposed before for the stabilization of wave equation, see [14] for the stabilization (only simple pole at $s=0$ is used), and [15] for disturbance rejection. While an exponential stability result has been given in [14], only asymptotic stability result has been given in [15]. A similar controller without any pole on the imaginary axis has been proposed for the beam equation in [13]. We then show that if $\omega_{1}$ does not belong to a countable set (e.g., the zeros of a transcendental function), then the closed loop system is asymptotically stable in general, and is exponentially stable in some special cases. We note that in many cases exponential stability is desired, due to, e.g., the robustness of the resulting closed-loop system, and in infinite dimensional systems, asymptotic stability may not imply exponential stability. We also relate this set of zeroes with the transmission zeroes of the appropriate transfer function. We then consider the case where the output of the controller is corrupted by disturbance. We show that if the structure of the disturbance is known (i.e., the frequency spectrum), then it may be possible to choose the controller accordingly to attenuate the effect of the disturbance at the system output.

This paper is organized as follows. In Section II, we introduce the system considered and propose a class of controller for stabilization. In Section III, we give some stability results. In Section IV, we consider disturbance rejection problem. Finally, we give some concluding remarks in Section V.

\section{Problem Statement}

We consider a flexible beam clamped at one end and is free at the other end. Without loss of generality, we assume that the beam length, mass density and the flexural rigidity are given as $L=1, \rho=1$ and $T=1$, respectively. We denote the displacement of the beam by $u(x, t)$ at $x \in(0,1)$ and $t \geq 0$. The beam is clamped at one end and is controlled by a boundary control force at the other end. The equations are given as $(x \in(0,1), t \geq 0)$

$$
\begin{gathered}
u_{t t}+u_{x x x x}=0, \\
u(0, t)=0, \quad u_{x}(0, t)=0 \\
u_{x x}(1, t)=0, \quad u_{x x x}(1, t)=f(t)
\end{gathered}
$$

where a subscript, as in $u_{t}$ denotes a partial differential with respect to the corresponding variable, and $f(\cdot): \mathbf{R}_{+} \rightarrow \mathbf{R}$ is the boundary control force applied at the free end of the beam. 
In this paper we assume that $f(t)$ is generated by a dynamic controller whose relation between its input $u_{t}(1, t)$, and its output $f(t)$ is given by the following:

$$
\hat{f}(s)=g(s) \hat{u}_{t}(1, s)
$$

where a hat denotes the Laplace transform of the corresponding variable. In (4), $g(s)$ is the controller transfer function, which is assumed to be a proper and rational function of $s$. We assume that the controller transfer function $g(s)$ has the following form:

$$
g(s)=g_{1}(s)+\frac{k}{s}+\frac{k_{1} s}{s^{2}+\omega_{1}^{2}},
$$

where $g_{1}(s)$ is a strictly positive-real function, and $k, k_{1}, \omega_{1} \in \mathbf{R}$.

In [3], a static controller with $g(s)=d>0$ was considered and it was shown that the closed-loop system is exponentially stable. The dynamic controller given by (5) with $k=k_{1}=0$ was considered in [13] for the beam equation and an exponential stability result was given. For the wave equation, in [14] the case $k_{1}=0$ was considered and an exponential stability result was given, and in [15] only an asymptotic stability result was given for the case $k_{1}>0$.

To analyze the well-posedness of the system given by (1)-(3), (4), we need a state-space representation for the controller given by (4) and (5). Let $(A, b, c, d)$ be a minimal (i.e., controllable and observable) representation of $g_{1}(s)$. Then, noting that the term $k$ actually corresponds to an integrator, we obtain the following state-space representation for the controller given by (4), (5):

$$
\begin{gathered}
\dot{z}_{1}=A z_{1}+b u_{t}(1, t) \\
\dot{x}_{1}=\omega_{1} x_{2}, \quad \dot{x}_{2}=-\omega_{1} x_{1}+u_{t}(1, t) \\
f(t)=c^{T} z_{1}+d u_{t}(1, t)+k u(1, t)+k_{1} x_{2}
\end{gathered}
$$

where $g_{1}(s)=c^{T}(s I-A)^{-1} b+d, z_{1} \in \mathbf{R}^{n}$, for some natural number $n$, is the actuator state, $A \in \mathbf{R}^{n \times n}$ is a constant matrix, $b, c \in \mathbf{R}^{n}$ are constant column vectors, $d \in \mathbf{R}$, and the superscript $T$ denotes transpose.

We make the following assumptions concerning the actuator given by (6), (8) throughout this work.

Assumption 1: All eigenvalues of $A \in \mathbf{R}^{n \times n}$ have negative real parts.

Assumption 2: $(A, b)$ is controllable and $(c, A)$ is observable.

Assumption 3: $d \geq 0, k \geq 0, k_{1} \geq 0$; moreover there exists a constant $\gamma, d \geq \gamma \geq 0$, such that the following holds:

$$
d+\mathcal{R} e\left\{c^{T}(j \omega I-A)^{-1} b\right\}>\gamma, \quad \omega \in \mathbf{R}
$$

where $g_{1}(s)=c^{T}(s I-A)^{-1} b+d$. Moreover for $d>0$, we assume $\gamma>0$ as well.

\section{StABILITY RESUlts}

Let the Assumptions 1-3 stated above hold. Then, since the transfer function $g_{1}(s)=d+c^{T}(s I-A)^{-1} b$ is strictly positive real it follows from the Meyer-Kalman-Yakubovich Lemma that given any symmetric positive definite matrix $Q \in \mathbf{R}^{n \times n}$, there exists a symmetric positive definite matrix $P \in \mathbf{R}^{n \times n}$, a vector $q \in \mathbf{R}^{n}$ and a constant $\epsilon>0$ satisfying (see [19, p. 133])

$$
\begin{aligned}
A^{T} P+P A & =-q q^{T}-\epsilon Q \\
P b-c & =\sqrt{2(d-\gamma)} q
\end{aligned}
$$

To analyze the system given by (1)-(3), (6)-(8), we first define the function space $\mathcal{H}$ as follows:

$$
\begin{aligned}
\mathcal{H}=\left\{\left(u v z_{1} x_{1} x_{2}\right)^{T} \mid u \in \mathbf{H}_{0}^{2}, v \in \mathbf{L}^{2},\right. & \\
& \left.z_{1} \in \mathbf{R}^{n}, x_{1}, x_{2} \in \mathbf{R},\right\}
\end{aligned}
$$

where the spaces $\mathbf{L}^{2}$, and $\mathbf{H}_{0}^{k}$ are defined as follows:

$$
\begin{aligned}
\mathbf{L}^{2} & =\left\{f:[0,1] \rightarrow \mathbf{R} \mid \int_{0}^{1} f^{2} d x<\infty\right\}, \\
\mathbf{H}^{k} & =\left\{f \in \mathbf{L}^{2} \mid f^{\prime}, \ldots, f^{(k)} \in \mathbf{L}^{2}\right\}, \\
\mathbf{H}_{0}^{k} & =\left\{f \in \mathbf{H}^{k} \mid f(0)=f^{\prime}(0)=0\right\} .
\end{aligned}
$$

The equations (1)-(3), (6)-(8) can be written in the following abstract form:

$$
\dot{z}=L z, \quad z(0) \in \mathcal{H}
$$

where $z=\left(u u_{t} z_{1} x_{1} x_{2}\right)^{T} \in \mathcal{H}$, the operator $L: \mathcal{H} \rightarrow \mathcal{H}$ is a linear unbounded operator defined as

$$
L\left(\begin{array}{c}
u \\
v \\
z_{1} \\
x_{1} \\
x_{2}
\end{array}\right)=\left(\begin{array}{c}
v \\
-u_{x x x x} \\
A z_{1}+b v(1) \\
\omega_{1} x_{2} \\
-\omega_{1} x_{1}+v(1)
\end{array}\right) .
$$

The domain $D(L)$ of the operator $L$ is defined as:

$$
\begin{aligned}
D(L)= & \left\{\left(u v z_{1} x_{1} x_{2}\right)^{T} \in \mathcal{H} \mid u \in \mathbf{H}_{0}^{4},\right. \\
& v \in \mathbf{H}_{0}^{2}, z_{1} \in \mathbf{R}^{n}, x_{1}, x_{2} \in \mathbf{R} ; \\
& u_{x x}(1)=0,-u_{x x x}(1)+c^{T} z_{1}+d v(1) \\
& \left.+k u(1)+k_{1} x_{2}=0\right\} .
\end{aligned}
$$

Let the Assumptions 1-3 hold, let $Q \in \mathbf{R}^{n \times n}$ be an arbitrary symmetric positive-definite matrix and let $P \in \mathbf{R}^{n \times n}, q \in \mathbf{R}^{n}$ be the solutions of (10) and (11) where $P$ is also a symmetric and positive definite matrix. In $\mathcal{H}$, we define the following "energy" inner-product:

$$
\begin{aligned}
\langle y, \tilde{y}\rangle_{E}=\frac{1}{2} \int_{0}^{1} v \tilde{v} d x+ & \frac{1}{2} \int_{0}^{1} u_{x x} \tilde{u}_{x x} d x+\frac{1}{2} k u(1) \tilde{u}(1) \\
& +\frac{1}{2}{\widetilde{z_{1}}}^{T} P z_{1}+\frac{1}{2} k_{1}\left(x_{1} \tilde{x}_{1}+x_{2} \tilde{x}_{2}\right)
\end{aligned}
$$

where $y=\left(u v z_{1} x_{1} x_{2}\right)^{T}, \tilde{y}=\left(\tilde{u} \tilde{v} \tilde{z}_{1} \tilde{x}_{1} \tilde{x}_{2}\right)^{T} \in \mathcal{H}$. It can be shown that $\mathcal{H}$, together with the energy inner-product given by (18) becomes a Hilbert space. The "energy" norm induced by (18) [for the solution $z(t)$ of (15)] is given by:

$$
\begin{aligned}
E(t)=\|z(t)\|^{2}= & \frac{1}{2} \int_{0}^{1} u_{t}^{2} d x+\frac{1}{2} \int_{0}^{1} u_{x x}^{2} d x \\
& +\frac{1}{2} k u^{2}(1, t)+\frac{1}{2} z_{1}^{T} P z_{1}+\frac{1}{2} k_{1}\left(x_{1}^{2}+x_{2}^{2}\right) .
\end{aligned}
$$

Theorem 1: Consider the system given by (15) with $d \geq 0, k \geq 0$ and $k_{1} \geq 0$.

i) The operator $L$ generates a $C_{0}$-semigroup of contractions $T(t)$ in $\mathcal{H}$, (for the terminology of semigroup theory, the reader is referred to, e.g., [17]).

ii) If $k_{1}>0$ and $\omega=\omega_{1}$ is not one of the roots of the following transcendental equation:

$$
\cosh \tau \sin \tau-\sinh \tau \cos \tau=0, \quad \tau=\sqrt{\omega}
$$


then the semigroup $T(t)$ generated by $L$ is asymptotically stable, that is all solutions of (15) asymptotically converge to zero.

Proof: i) We use Lumer-Phillips theorem, to prove the assertion i), see, e.g., [17]. To prove that $L$ is dissipative, we compute $\langle y, L y\rangle_{E}$ by using (16) in (18). Then, integrating by parts, and using (10), (11), (14), (17) we obtain

$$
\begin{aligned}
\langle y, L y\rangle_{E}=-\frac{\gamma}{2} v^{2}(1) & -\frac{1}{4} \\
& {\left[\sqrt{2(d-\gamma)} v(1)-z_{1}^{T} q\right]^{2}-\frac{\epsilon}{4} z_{1}^{T} Q z_{1} . }
\end{aligned}
$$

Since $\langle y, L y\rangle_{E} \leq 0$, it follows that $L$ is dissipative, (see [14] for similar calculations).

After some straightforward calculations, it can be easily shown that $\lambda I-L: \mathcal{H} \rightarrow \mathcal{H}$ is onto for $\lambda>0$, (see [14] for similar calculations). Then, it follows from the Lumer-Phillips theorem that $L$ generates a $C_{0}$-semigroup of contractions $T(t)$ on $\mathcal{H}$.

ii) To prove the assertion ii), we use LaSalle's invariance principle, extended to infinite dimensional systems, see [18] and [10]. According to this principle, all solutions of (15) asymptotically tend to the maximal invariant subset of the following set:

$$
\mathcal{S}=\{z \in \mathcal{H} \mid \dot{E}=0\}
$$

provided that the solution trajectories for $t \geq 0$ are precompact in $\mathcal{H}$. Since the operator $L: \mathcal{H} \rightarrow \mathcal{H}$ generates a $C_{0}$-semigroup of contractions on $\mathcal{H}$ (hence, the solution trajectories are bounded on $\mathcal{H}$ for $t \geq$ 0 ), the precompactness of the solution trajectories are guaranteed if the operator $(\lambda I-L)^{-1}: \mathcal{H} \rightarrow \mathcal{H}$ is compact for some $\lambda>0$, see [10]. To prove the last property, we first show that $L^{-1}$ exists and is a compact operator on $\mathcal{H}$. To see this, let $q=\left(f h r r_{1} r_{2}\right)^{T} \in \mathcal{H}$ be given. We want to solve the equation $L z=q$ for $z$, where $z=\left(u v z_{1} x_{1} x_{2}\right)^{T} \in D(L)$. The solution of this equation can easily be found as:

$$
\begin{aligned}
u(x)= & -\int_{0}^{x} \int_{0}^{\xi_{3}} \int_{0}^{\xi_{2}} \int_{0}^{\xi_{1}} h(\sigma) d \sigma d \xi_{1} d \xi_{2} d \xi_{3} \\
& +c_{1} x^{3}+c_{2} x^{2}, \quad v(x)=f(x) \\
z_{1}= & A^{-1}(r-f(1) b), \quad x_{1}=\frac{f(1)-r_{2}}{\omega_{1}}, \quad x_{2}=\frac{r_{1}}{\omega_{1}}
\end{aligned}
$$

where the constants $c_{1}, c_{2}$ can be uniquely determined from (8). It follows that $L^{-1}$ exists and maps $\mathcal{H}$ into $\mathbf{H}^{4} \times \mathbf{H}^{2} \times \mathbf{R}^{n} \times \mathbf{R} \times \mathbf{R}$, moreover $\left(u v z_{1} x_{1} x_{2}\right)^{T} \in D(L)$. Since $q=\left(f h r r_{1} r_{2}\right)^{T} \in \mathcal{H}$ it follows that $f \in \mathbf{H}_{0}^{2}$, see (12). Hence, if $\|q\|$ is bounded in $\mathcal{H}$, it follows easily that that $f(1)$ is bounded as well. Therefore $L^{-1}$ maps the bounded sets of $\mathcal{H}$ into the bounded sets of $\mathbf{H}^{4} \times \mathbf{H}^{2} \times \mathbf{R}^{n} \times \mathbf{R} \times \mathbf{R}$. Since the embedding of the latter into $\mathcal{H}$ is compact, see [20, p. 14], it follows that $L^{-1}$ is a compact operator. This also proves that the spectrum of $L$ consists entirely of isolated eigenvalues, and that for any $\lambda$ in the resolvent set of $L$, the operator $(\lambda I-L)^{-1}: \mathcal{H} \rightarrow \mathcal{H}$ is a compact operator, see [9, p. 187]. Furthermore, our argument above shows that $\lambda=0$ is not an eigenvalue of $L$. Since the operator $L$ generates a $C_{0}$-semigroup of contractions on $\mathcal{H}$, by the argument given above it follows that the solutions trajectories of (15) are precompact in $\mathcal{H}$ for $t \geq 0$, hence by LaSalle's invariance principle, the solutions asymptotically tend to the maximal invariant subset of $\mathcal{S}$ [see (22)]. Hence, to prove that all solutions of (15) asymptotically tend to the zero solution, it suffices to show that $\mathcal{S}$ contains only the zero solution, which is a typical procedure in the application of LaSalle's invariance principle.

By using (15) and (19), we see that $\dot{E}=2\langle z, L z\rangle_{E}$. Hence, from (21) we obtain

$$
\begin{aligned}
\dot{E}=2\langle z, L z\rangle_{E} & =-\gamma u_{t}^{2}(1, t) \\
- & \frac{1}{2}\left[\sqrt{2(d-\gamma)} u_{t}(1, t)-z_{1}^{T} q\right]^{2}-\frac{\epsilon}{2} z_{1}^{T} Q z_{1} .
\end{aligned}
$$

To prove that $\mathcal{S}$ contains only the zero solution, we set $\dot{E}=0$ in (25), which results in $z_{1}=0$. This implies that $\dot{z}_{1}=0$, hence by using (6) and (8) we obtain $u_{t}(1, t)=0, f(t)=k u(1, t)+k_{1} x_{2}$. Hence, all solutions of (15) in $\mathcal{S}$ satisfy the following:

$$
\begin{aligned}
& u_{t t}+u_{x x x x}=0, \quad \dot{x}_{1}=\omega_{1} x_{2}, \quad \dot{x}_{2}=-\omega_{1} x_{1} \\
& u(0, t)=0, \quad u_{x}(0, t)=0, \\
& u_{x x}(1, t)=0, \quad u_{t}(1, t)=0 \\
& u_{x x x}(1, t)=k u(1, t)+k_{1} x_{2} .
\end{aligned}
$$

Consider the system given by (26), (27). This system can be put into the form $\dot{z}_{p}=L_{p} z_{p}$ with $z_{p}=\left(u u_{t} x_{1} x_{2}\right)^{T} \in \mathcal{H}_{p}$, where $\mathcal{H}_{p}$ is the same as given by (12) with obvious omission of $z_{1}$, and $L_{p}$ is similar to $L$ given by (16) with obvious omission of $z_{1}$ (i.e., the third row) and $v(1)=0 . D\left(L_{p}\right)$ is given as (17) with the omission of $z_{1}$, and the last boundary condition should be replaced by $v(1)=0 . \mathcal{H}_{p}$ is also a Hilbert space with the inner-product $\langle\cdot, \cdot\rangle_{p}$ induced by (18) with the omission of $z_{1}$. By straightforward calculation, and using integration by parts, it can easily be shown that $\left\langle z_{p}, L_{p} \tilde{z}_{p}\right\rangle_{p}=-\left\langle L_{p} z_{p}, \tilde{z}_{p}\right\rangle_{p}$, for any $z_{p}, \tilde{z}_{p} \in D\left(L_{p}\right)$, hence from [5, Th. 4.1], it follows that $L_{p}$ is skew-adjoint, i.e., $B_{p}=j L_{p}$ is self-adjoint. Hence, it follows from [17, Th. 10.8] that $L_{p}$ generates a $C_{0}$ semigroup of contractions. Also, by using [5, Th. 5.1], it follows that there exists a complete set of orthonormal basis $\left\{\varphi_{1}, \varphi_{2}, \ldots\right\}$ on $\mathcal{H}_{p}$ consisting of eigenvectors of $L_{p}$. Moreover, for any $z_{0} \in D\left(L_{p}\right)$, we have $z_{0}=\sum_{j}\left\langle z_{0}, \varphi_{j}\right\rangle_{p} \varphi_{j}$ and $L_{p} z_{0}=\sum_{j} \lambda_{j}\left\langle z_{0}, \varphi_{j}\right\rangle_{p} \varphi_{j}$, where $\lambda_{j}$ denotes the eigenvalues of $L_{p}$, see [5, Th. 5.1]. Hence, the solution $z_{p}(t)$ of (26), (27) can be given as $z_{p}(t)=\sum_{j} c_{j} e^{\lambda_{j} t} \varphi_{j}$, where the coefficients $c_{j} \in \mathbf{C}$ can be determined from initial conditions and the eigenvectors $\varphi$ have the form $\varphi=\left(u v x_{1} x_{2}\right)^{T} \in D\left(L_{p}\right)$. Due to the structure of $L_{p}, \lambda= \pm j \omega_{1}$ is an eigenvalue pair, and since $\tau=\sqrt{\omega_{1}}$ is not a root of (20), the corresponding eigenvectors have $u=v=0$. The rest of the eigenvalues have the form $\lambda= \pm j \tau^{2}$, where $\tau$ is a root of (20), and since we have $\tau \neq \sqrt{\omega_{1}}$, the corresponding eigenvectors have $x_{1}=x_{2}=0$ with $u(0)=u^{\prime}(0)=u^{\prime \prime}(1)=v(1)=0$. By using these in (28), after some straightforward calculations, we obtain $c_{j}=0, j=1,2, \ldots$ Hence, $z_{p}=0$ is the only possible solution of (26)-(28). Hence, by LaSalle's invariance theorem, we conclude that the solutions of (15) asymptotically tend to the zero solution.

Theorem 1 remains valid even if $k_{1}=0$, provided that the variables $x_{1}$ and $x_{2}$ are suppressed everywhere. The proof of this fact is essentially the same as the proof of Theorem 1 .

It was proven in [13] that for $k=k_{1}=0$, if $d>0$, then the closed-loop system (1)-(3), (6) and (8) is exponentially stable. Since the subsystem (7) is essentially finite dimensional, we may expect the same conclusions hold for the case $k \geq 0, k_{1}>0$ as well. In the sequel we will prove this result by using Huang's Theorem stated below:

Theorem (Huang): Let $L$ be a linear operator on a Hilbert space $\mathcal{H}$. Assume that $L$ generates a bounded $C_{0}$ semigroup $T(t)$ on $\mathcal{H}$. Then, $T(t)$ is exponentially stable if and only if the following holds:

i) imaginary axis belongs to the resolvent set of $L$.

ii) the following resolvent estimate holds:

$$
\sup _{\omega \in \mathbf{R}}\left\|(j \omega I-L)^{-1}\right\|<\infty .
$$

Proof: See [6] or [10] for an alternative proof.

In the sequel, we will work on the complexified versions of the Hilbert spaces mentioned above; for convenience we do not change the notation.

Theorem 2: Consider the system given by (15) with $d>0, k \geq 0$ and $k_{1}>0$. Let the assumptions $1-3$ hold. If $\omega=\omega_{1}$ is not a root of (20), then the semigroup $T(t)$ generated by $L$ is exponentially stable.

Proof: We will use Huang's theorem cited above. First note that by Theorem 1, $T(t)$ is bounded. If $\tau=\sqrt{\omega_{1}}$ is not a root of (20), then by the part ii) of Theorem 1, the semigroup $T(t)$ generated by $L$ is asymptotically stable, hence $L$ cannot have an eigenvalue on the 
imaginary axis, and since $L$ has compact resolvent, it follows that the imaginary axis belongs to the resolvent set of $L$, see, e.g., [10, Th. 3.26].

Next, we will show that the resolvent estimate given by (29) holds for sufficiently large $\omega$. The calculations are lengthy, but straightforward, similar to the ones given in [4]. Here we will omit the details, and give only basic steps of the calculations. Let $y=\left(p q r r_{1} r_{2}\right)^{T} \in \mathcal{H}$ and $\lambda$, in the resolvent set of $L$, be given and let $z=\left(u v z_{1} x_{1} x_{2}\right)^{T} \in D(L)$ be the solution of the following:

$$
(\lambda I-L) z=y
$$

In the sequel, we will use $\lambda=j \omega, \omega \in \mathbf{R}$, for simplicity. The solution $u$ of (30) satisfying $u(0)=u_{x}(0)=0$ is given by

$$
\begin{aligned}
u(x)= & A(\cosh \tau x-\cos \tau x)+B(\sinh \tau x-\sin \tau x) \\
+ & \frac{1}{2 \tau^{3}} \int_{0}^{x}[\sinh \tau(x-\sigma)-\sin \tau(x-\sigma)] \\
& \cdot[\lambda p(\sigma)+q(\sigma)] d \sigma, \quad \tau=\sqrt{\omega}
\end{aligned}
$$

where $A$ and $B$ are determined by the remaining boundary conditions

$$
\begin{array}{r}
u_{x x}(1)=0, \quad u_{x x x}(1) \\
-\lambda g(\lambda) u(1)=\left(\frac{k}{\lambda}-g(\lambda)\right) p(1) \\
+k_{1} \frac{\lambda r_{2}-\omega_{1} r_{1}}{\lambda^{2}+\omega_{1}^{2}}+c^{T}(\lambda I-A)^{-1} r
\end{array}
$$

and $g(\cdot)$ is given by (5). Using (31) in (32) we obtain

$$
\begin{aligned}
A= & \frac{1}{\Delta}\left\{\left[\cosh \tau+\cos \tau-\frac{\lambda g(\lambda)}{\tau^{3}}(\sinh \tau-\sin \tau)\right]\right. \\
& \left.\cdot X_{1}-(\sinh \tau+\sin \tau)\left(X_{2}+X_{3}+X_{4}\right)\right\} \\
B= & \frac{1}{\Delta}\left\{\left[-\sinh \tau+\sin \tau+\frac{\lambda g(\lambda)}{\tau^{3}}(\cosh \tau-\cos \tau)\right]\right. \\
& \left.\cdot X_{1}+(\cosh \tau+\cos \tau)\left(X_{2}+X_{3}+X_{4}\right)\right\}
\end{aligned}
$$

where

$$
\begin{aligned}
\Delta= & 2\left(1+\cosh \tau \cos \tau-\frac{\lambda g(\lambda)}{\tau^{3}}\right. \\
& (\sinh \tau \cos \tau-\cosh \tau \sin \tau)) \\
X_{1}= & -\frac{1}{2 \tau^{3}} \int_{0}^{1}[\sinh \tau(1-\sigma)+\sin \tau(1-\sigma)] \\
& \cdot[\lambda p(\sigma)+q(\sigma)] d \sigma \\
X_{2}= & \frac{1}{\tau^{3}}\left(\frac{k}{\lambda}-g(\lambda)\right) p(1)+\frac{k_{1}}{\tau^{3}} \frac{\lambda r_{2}-\omega_{1} r_{1}}{\lambda^{2}+\omega_{1}^{2}} \\
& +\frac{c^{T}(\lambda I-A)^{-1} r}{\tau^{3}} \\
X_{3}= & \frac{1}{2 \tau^{3}} \int_{0}^{1}[\cosh \tau(1-\sigma)+\cos \tau(1-\sigma)] \\
& \cdot[\lambda p(\sigma)+q(\sigma)] d \sigma \\
X_{4}= & \frac{1}{2 \tau^{3}} \frac{\lambda g(\lambda)}{\tau^{3}} \int_{0}^{1}[\sinh \tau(1-\sigma)-\sin \tau(1-\sigma)] \\
& \cdot[\lambda p(\sigma)+q(\sigma)] d \sigma .
\end{aligned}
$$

(Note that $\Delta=0$ may be considered as the characteristic equation in the sense that its roots are the eigenvalues of the closed-loop system.) In the sequel, by using estimates of (33)-(39), we will show that an estimate of the form $\|z\| \leq M\|y\|$ holds. We will use the following notation: for any function $F(\cdot)>0, \mathcal{O}(F(\tau))$ denotes any function which satisfies $\mathcal{O}(F(\tau)) \leq M F(\tau)$ for some $M>0$ and for $\tau$ sufficiently large. Also, for any $f \in \mathbf{L}^{2},\|f\|_{2}$ denotes the $\mathbf{L}^{2}$ norm of $f$.

For simplicity, assume that $\tau>0$. Using integration by parts in (36), (38) and (39), we obtain

$$
\begin{aligned}
X_{i}= & -\frac{1}{4 \tau^{3}} e^{\tau} \int_{0}^{1} e^{-\tau \sigma}\left[j p_{x x}(\sigma)+q(\sigma)\right] d \sigma \\
& +\mathcal{O}\left(\frac{\left\|p_{x x}\right\|_{2}+\|q\|_{2}}{\tau^{3}}\right), \quad i=1,3 \\
X_{4}= & \frac{j g(\lambda)}{\tau^{3}} p(1)+\frac{\lambda g(\lambda)}{4 \tau^{6}} e^{\tau} \int_{0}^{1} e^{-\tau \sigma}\left[j p_{x x}(\sigma)+q(\sigma)\right] d \sigma \\
& +\mathcal{O}\left(\frac{\left\|p_{x x}\right\|_{2}+\|q\|_{2}}{\tau^{4}}\right) .
\end{aligned}
$$

Let $\hat{u}$ denote the integral term in (31). Upon differentiating and using integration by parts, we obtain

$$
\begin{aligned}
\hat{u}_{x x}(x)=\frac{1}{4 \tau} e^{\tau x} \int_{0}^{1} e^{-\tau \sigma}\left[j p_{x x}(\sigma)\right. & +q(\sigma)] d \sigma \\
& +\mathcal{O}\left(\frac{\left\|p_{x x}\right\|_{2}+\|q\|_{2}}{\tau}\right) .
\end{aligned}
$$

In (35), by collecting dominant terms, we obtain

$$
\Delta=2+e^{\tau}\left(\cos \tau-\frac{j g(\lambda)}{\tau}(\cos \tau-\sin \tau)\right)+\mathcal{O}\left(e^{-\tau}\right) .
$$

Let $g(\lambda)=R(\omega)+j I(\omega)$ where $R$ and $I$ denote the real and imaginary parts, respectively. Moreover we have $R(\omega) \geq \gamma>0$ and $I(\omega)=$ $\mathcal{O}\left(1 / \tau^{2}\right)$ for large $\tau$. Noting that $\cos ^{2} \tau+(\cos \tau-\sin \tau)^{2} \geq c>0$ for some $c$, from (43) we obtain $\Delta=\mathcal{O}\left(e^{\tau} / \tau\right)$ for large $\tau$.

By using (33), (34), (40), (41) and (42) in (31) and noting that $\Delta^{-1}=\mathcal{O}\left(\tau e^{-\tau}\right)$, after straightforward algebraic calculations we obtain the following estimate for $\tau$ sufficiently large:

$$
\left\|u_{x x}\right\|_{2} \leq M_{1}\|y\|
$$

where $M_{1}>0$ is a constant and the norm $\|\cdot\|$ is given by (19). Note that in this calculation the key point is the fact that the dominant terms in (40)-(42), which are the integral terms, cancel in the expression of $u_{x x}$, see [4] for a similar result. By using (33), (34), (40), (41), (42) and (31) in $v=\lambda u-p$, [see (30)], after straightforward calculations similar to the ones mentioned above we obtain the following estimate for $\tau$ sufficiently large:

$$
\|v\|_{2} \leq M_{2}\|y\|
$$

where $M_{2}>0$ is a constant. Similarly, from (30) we obtain

$$
\begin{aligned}
\left\|z_{1}\right\|_{n} & =\mathcal{O}\left(\frac{|v(1)|+\|r\|_{n}}{\tau^{2}}\right) \\
\left|x_{i}\right| & =\mathcal{O}\left(\frac{|v(1)|+\left|r_{1}\right|+\left|r_{2}\right|}{\tau^{2}}\right), \quad i=1,2
\end{aligned}
$$

where $\|\cdot\|_{n}$ denotes the norm in $\mathbf{R}^{n}$, see (19). Finally. note that $v(1)=$ $\lambda u(1)-p(1)$, and $|u(1)| \leq\left\|u_{x x}\right\|_{2},|p(1)| \leq\left\|p_{x x}\right\|_{2}$. Combining these, we obtain the following inequality for sufficiently large $\tau$ :

$$
\|z\| \leq M\|y\|
$$

where $M>0$ is a constant, and for a given $y \in \mathcal{H}, z \in D(L)$ is the solution of (30). Hence for some $\Omega>0$ sufficiently large we have

$$
\sup _{\omega \geq \Omega}\left\|(j \omega I-L)^{-1}\right\|<\infty .
$$


Since $L$ has compact resolvent and the imaginary axis is in the resolvent set, we also have the following:

$$
\sup _{\omega \leq \Omega}\left\|(j \omega I-L)^{-1}\right\|<\infty .
$$

Combining (48) and (49) we obtain (29). Hence, by Huang's Theorem, $L$ generates an exponentially stable semigroup in $\mathcal{H}$.

Theorem 2 remains valid even if $k_{1}=0$, provided that the variables $x_{1}$ and $x_{2}$ are suppressed everywhere. The proof of this fact is essentially the same as the proof of Theorem 2 .

\section{Disturbance REJECTION}

In this section, we show the effect of the proposed control law given by (6)-(8) on the solutions of the system given by (1)-(3), when the output of the controller is corrupted by a disturbance $n(t)$, that is (8) has the following form:

$$
f(t)=c^{T} z_{1}+d u_{t}(1, t)+k u(1, t)+k_{1} x_{2}+n(t)
$$

or, equivalently, (4) has the following form:

$$
\hat{f}(s)=g(s) \hat{y}_{t}(1, s)+\hat{n}(s)
$$

where $\hat{n}(s)$ is the Laplace transform of the disturbance $n(t)$ and $g(s)$ is given by (5).

To find the transfer function from $n(t)$ to $u_{t}(1, t)$, first we need to find the transfer function from $f(t)$ to $u_{t}(1, t)$. By taking the Laplace transform of (1)-(3) and using zero initial conditions, after some straightforward calculations we obtain the following:

$$
\begin{aligned}
\hat{u}_{t}(1, s) & =-h(s) \hat{f}(s), \\
h(s) & =j \frac{(\cosh \tau \sin \tau-\sinh \tau \cos \tau)}{\tau(1+\cosh \tau \cos \tau)}
\end{aligned}
$$

where $s^{2}=-\tau^{4}$, see also [1]. By using (52) in (51), we obtain

$$
\hat{u}_{t}(1, s)=-\frac{h(s)}{1+h(s) g(s)} \hat{n}(s) .
$$

Remark 1: Consider the system given by (1)-(3). If we consider $f$ as an input and $u_{t}(1, t)$ as output, it is known that this system is passive, see [10]; moreover the transfer function of this system is given by $h(s)$ in (52). On the other hand, the controller transfer function $g(s)$ which is given by (5) is MSPR, see [7]. It was shown in [7] that for finite-dimensional linear, time-invariant systems (LTI), in the classical negative feedback configuration if $h(s)$ (the system to be controlled) is positive real and if $g(s)$ (the controller) is MSPR, then the closed-loop system is asymptotically stable, provided that none of the imaginary axis poles of $g(s)$ is a transmission zero of $h(s)$. Note that this result may not hold for infinite dimensional systems. In our case, (1)-(3) represent a passive system, which is equivalent to positive realness in finite dimensional LTI systems. It can easily be shown that $\omega=\omega_{1}$ is a root of (20) if and only if $h\left(j \omega_{1}\right)=0$. In this sense, Theorem 2 may be considered as an extension of the stated result of [7] to an infinite dimensional system given by (1)-(3). Note that, although in finite dimensional LTI case the asymptotic stability implies exponential stability, this is not necessarily true in infinite dimensional systems. We also note that a similar result holds for the wave equation, [16].

Remark 2: The controller given by (5) can easily be generalized to

$$
g(s)=g_{1}(s)+\frac{k}{s}+\sum_{i=1}^{N} \frac{k_{i} s}{s^{2}+\omega_{i}^{2}}
$$

for any $N$. Theorem 2 will remain valid, provided that $k_{i} \geq 0$ and $\omega_{i}$ is not a root of (20) for $i=1,2, \ldots, N$.

From (53), we can also derive a procedure to design $g(s)$ if we know the structure of $n(t)$. For example if $n(t)$ has a band-limited frequency spectrum, (i.e., has frequency components in an interval of frequencies $\left.\left[\Omega_{1}, \Omega_{2}\right]\right)$, then we can choose $g(s)$ to minimize

$$
c(\omega)=\left|\frac{h(j \omega)}{1+h(j \omega) g(j \omega)}\right|, \quad \omega \in\left[\Omega_{1}, \Omega_{2}\right] .
$$

Note that to ensure the stability of the closed-loop system, $g_{1}(s)$ should be a strictly positive real function as well, [see (5)]. As a simple example, assume that $n(t)=a \cos \omega_{0}(t)$. Then we may choose $g(s)$ in the form (5) with $\omega_{1}=\omega_{0}$. Provided that the Assumptions 1-3 are satisfied and that $j \omega_{0}$ is not a zero of $h(s)$, the closed-loop system is asymptotically stable, (see Theorem 1 ). Moreover, if $k_{1}>0$, then $c(\omega)$ given above satisfies $c\left(\omega_{0}\right)=0$. From (55), we may conclude that this eliminates the effect of the disturbance at the output $u_{t}(1, t)$.

\section{CONCLUSION}

In this note, we considered a linear time invariant system which is represented by one-dimensional Euler-Bernoulli beam equation in a bounded domain. We assumed that the system is clamped at one end and the boundary control force input is applied at the other end. For this system, we proposed a finite dimensional dynamic boundary controller. This introduces extra degrees of freedom in designing controllers which could be exploited in solving a variety of control problems, such as disturbance rejection, pole assignment, etc., while maintaining stability. The transfer function of the controller is a proper rational function of the complex variable $s$, and may contain a single pole at $s=0$ and another one $s=j \omega_{1}, \omega_{1} \neq 0$, provided that the residues corresponding to these poles are nonnegative; the rest of the transfer function is required to be a strictly positive real function. We then proved that the closed-loop system is asymptotically stable provided that $s=j \omega_{1}$ is not a zero of an appropriate system transfer function, and is exponentially stable in some cases. We also studied the case where the output of the controller is corrupted by a disturbance. We showed that, if the frequency spectrum of the controller is known, then by choosing the controller appropriately we can obtain better disturbance rejection.

\section{REFERENCES}

[1] J. Bontsema and S. A. de Vries, "Robustness of flexible structures against small delays," in Proc. 27th Conf. Decision Control, Austin, TX, 1988, pp. 1647-1648.

[2] G. Chen, "Energy decay estimates and exact boundary value controllability for the wave equation in a bounded domain," J. Math. Pures. Appl., vol. 58, pp. 249-273, 1979.

[3] G. Chen, M. C. Delfour, A. M. Krall, and G. Payre, "Modeling, stabilization and control of serially connected beams," SIAM J. Control Optim., vol. 25, pp. 526-546, 1987.

[4] G. Chen, S. G. Krantz, D. W. Ma, and C. E. Wayne, "The Euler-Bernoulli beam equation with bending energy dissipation," in Operator Methods for Optimal Control Problems, S. J. Lee, Ed. New York: Marcel Dekker, 1987, pp. 67-96.

[5] I. Gohberg, S. Goldberg, and M. A. Kaashoek, Classes of Linear Operators. Basel, Germany: Birkhauser, 1990, vol. 1.

[6] F. L. Huang, "Characteristic conditions for exponential stability of linear dynamical systems in Hilbert spaces," Annal. of Diff. Eqns., vol. 1, pp. 43-53, 1985.

[7] S. M. Joshi and S. Gupta, "On a class of marginally stable positive real systems," IEEE Trans. Automat. Contr., vol. 41, pp. 152-155, Jan. 1996.

[8] J. Lagnese, "Decay of solutions of wave equations in a bounded domain with boundary dissipation," J. Differential Equations, vol. 50, pp. 163-182, 1983.

[9] T. Kato, Perturbation Theory for Linear Operators, 2nd ed. New York: Springer-Verlag, 1980. 
[10] Z. H. Luo, B. Z. Guo, and Ö. Morgül, Stability and Stabilization of Infinite Dimensional Systems With Applications, ser. Communications and Control Eng.. London, U.K.: Springer-Verlag, 1999.

[11] Ö. Morgül, "Control and stabilization of a flexible beam attached to a rigid body," Int. J. Control, vol. 51, pp. 11-33, 1990.

[12] _ - "Orientation and stabilization of a flexible beam attached to a rigid body: Planar motion," IEEE Trans. Automat. Contr., vol. 36, pp. 953-963, Aug. 1991.

[13] _ "Dynamic boundary control of a Euler-Bernoulli beam," IEEE Trans. Automat. Contr., vol. 37, pp. 639-642, May 1992.

[14] _ "A dynamic boundary control for the wave equation," Automatica vol. 30, no. 11, pp. 1785-1792, Oct. 1994.

[15] _ _Stabilization and disturbance rejection for the wave equation," IEEE Trans. Automat. Contr., vol. 43, pp. 89-95, Jan. 1998.

[16] — - "An exponential stability result for the wave equation," Automatica, submitted for publication

[17] A. Pazy, Semigroups of Linear Operators and Applications to Partial Differential Equations. New York: Springer-Verlag, 1983.

[18] S. H. Saperstone, Semidynamical Systems in Infinite Dimensional Systems. New York: Springer-Verlag, 1981.

[19] J. J. E. Slotine and W. Pi, Applied Nonlinear Control. Upper Saddle River, NJ: Prentice-Hall, 1991

[20] H. Tanabe, Equations of Evolution. New York: Pitman, 1979.

\section{Robust $\boldsymbol{H}_{\infty}$ Control of Discrete-Time Markovian Jump Linear Systems With Mode-Dependent Time-Delays}

\author{
E. K. Boukas and Z. K. Liu
}

\begin{abstract}
This note considers the class of discrete-time Markovian jump linear system with norm-bounded uncertainties and time-delay, which is dependent on the system mode. Linear matrix inequality (LMI) -based sufficient conditions for the stability, stabilization and $H_{\infty}$ control are developed. A numerical example is worked out to show the usefulness of the theoretical results.
\end{abstract}

Index Terms-Discrete-time Markovian jump linear system, $H_{\infty}$ control, linear matrix inequality (LMI), time-delay system.

\section{INTRODUCTION}

Discrete-time Markovian jump linear system is a hybrid one with state comprised of two components: a discrete part denoted by $r_{t}$ and a continuous part, denoted by $x_{t}$. Discrete state $r_{t}$ is a discrete-time Markov chain representing the mode of the system and $x_{t}$ denotes the physical state of the system, e.g., the inventory level in manufacturing systems. The continuous state $x_{t}$ evolves according to a difference equation when the mode is fixed. For more information on discrete-time Markovian jump linear systems, the reader is referred to [5], [6], and the references therein.

Time-delay occurs frequently in many practical systems, such as manufacturing system, telecommunication and economic systems etc., which is an important source of instability and poor performance. For continuous-time Markovian jump linear systems with time-delay, we refer the reader to [4]. For discrete-time Markovian jump linear system with time-delay, [1] studied the robust stability, stabilization and $H_{\infty}$ problem. The purpose of this note is to extend the results in [1] to the

Manuscript received January 19, 2001; revised June 11, 2001. Recommended by Associate Editor Q. Zhang.

The authors are with the Mechanical Engineering Department, École Polytechnique de Montréal, Montréal, QC, H3C 3A7 Canada.

Publisher Item Identifier S 0018-9286(01)11108-6. case where the time-delay in the system is dependent on the system mode.

The goal of this note is to study the robust stability and robust stabilizability of the class of discrete-time Markovian jump linear systems with time-delay and norm bounded uncertainties. The robust $H_{\infty}$ control is also considered. The sufficient conditions we will establish are all in linear matrix inequality (LMI) formalism which makes their resolution easy. The rest of this note is organized as follows: Section II describes the system model. Section III addresses the robust stability and stabilization problem. Section IV studies the robust $H_{\infty}$ control problem. Section $\mathrm{V}$ provides a numerical example to show the validness of the proposed results.

\section{MODEL DESCRIPTION}

Let $\left\{r_{k}, k \geq 0\right\}$ be a Markov chain with state space $\mathcal{S}=\{1, \ldots, N\}$ and state transition matrix $P=\left[p_{i j}\right]_{i, j \in \mathcal{S}}$, i.e., the transition probabilities of $\left\{r_{k}, k \geq 0\right\}$ are as follows:

$$
P\left[r_{k+1}=j \mid r_{k}=i\right]=p_{i j}, \quad \forall i, j \in \mathcal{S}
$$

with $p_{i j} \geq 0, \forall i, i \in \mathcal{S}$ and $\sum_{j=1}^{N} p_{i j}=1$, for $i \in \mathcal{S}$.

Consider a discrete-time hybrid system with $N$ modes. Suppose that the system mode switching is governed by $\left\{r_{k}, k \geq 0\right\}$ and the system parameters contain norm-bounded uncertainties. Let the system dynamics be described by the following:

$$
\left\{\begin{array}{l}
x_{t+1}=A\left(t, r_{t}\right) x_{t}+A_{d}\left(t, r_{t}\right) x_{t-\tau\left(r_{t}\right)} \\
\quad+B\left(r_{t}, t\right) u_{t}+B_{1}\left(r_{t}\right) w_{t}, \\
x_{s}=\alpha_{s}, s=-\tau, \ldots,-1, \\
z_{t}=C\left(r_{t}, t\right) x_{t}+C_{d}\left(r_{t}, t\right) x_{t-\tau\left(r_{t}\right)} \\
\quad+B_{c}\left(r_{t}, t\right) u(t)+C_{1}\left(r_{t}\right) w_{t}
\end{array}\right.
$$

where $x_{t} \in \mathbb{R}^{n}$ is the state of the system, for each $r_{t} \in \mathcal{S}$

$$
\begin{gathered}
A\left(r_{t}, t\right)=A\left(r_{t}\right)+\Delta_{a}\left(r_{t}, t\right) \\
A_{d}\left(r_{t}, t\right)=A_{d}\left(r_{t}\right)+\Delta_{d}\left(r_{t}, t\right) \\
B\left(r_{t}, t\right)=B\left(r_{t}\right)+\Delta_{b}\left(r_{t}, t\right) \\
C\left(r_{t}, t\right)=C\left(r_{t}\right)+\Delta_{c}\left(r_{t}, t\right) \\
C_{d}\left(r_{t}, t\right)=C_{d}\left(r_{t}\right)+\Delta_{c d}\left(r_{t}, t\right) \\
B_{c}\left(r_{t}, t\right)=B_{c}\left(r_{t}\right)+\Delta_{b c}\left(r_{t}, t\right)
\end{gathered}
$$

with $A\left(r_{t}\right), A_{d}\left(r_{t}\right), B_{1}\left(r_{t}\right), B\left(r_{t}\right), C\left(r_{t}\right), C_{d}\left(r_{t}\right), B_{c}\left(r_{t}\right)$ and $C_{1}\left(r_{t}\right)$ are matrices with appropriate dimensions, $\Delta_{a}\left(r_{t}, t\right)$, $\Delta_{d}\left(r_{t}, t\right), \Delta_{a}\left(r_{t}, t\right), \Delta_{b}\left(r_{t}, t\right), \Delta_{c}\left(r_{t}, t\right), \Delta_{c d}\left(r_{t}, t\right), \Delta_{b c}\left(r_{t}, t\right)$ are unknown matrices denoting the uncertainties in the system. $\tau\left(r_{t}\right)$ is a constant, denoting the time-delay of the system when the system is in mode $r_{t}$.

In this note, we assume that the admissible uncertainties satisfy the following:

$$
\begin{aligned}
\left(\begin{array}{ccc}
\Delta_{a}\left(r_{t}, t\right) & \Delta_{d}\left(r_{t}, t\right) & \Delta_{b}\left(r_{t}, t\right) \\
\Delta_{c}\left(r_{t}, t\right) & \Delta_{c d}\left(r_{t}, t\right) & \Delta_{b c}\left(r_{t}, t\right)
\end{array}\right) \\
\quad=\left(\begin{array}{l}
G_{1}\left(r_{t}\right) \\
G_{2}\left(r_{t}\right)
\end{array}\right) \Delta\left(r_{t}, t\right)\left(\begin{array}{lll}
H_{1}\left(r_{t}\right) & H_{2}\left(r_{t}\right) & \left.H_{3}\left(r_{t}\right)\right)
\end{array}\right.
\end{aligned}
$$

with $\Delta^{\top}\left(r_{t}, t\right) \Delta\left(r_{t}, t\right) \leq I, \forall r_{t} \in \mathbf{S}$. In the sequel, notation $X>$ $0(\geq 0)$, with $X$ being a matrix, means that $X$ is symmetric and (semi-) positive-definite. 